\title{
Study of Medium Voltage Electric Bridge Set of the Underground Coal Mine
}

\author{
Chen Wei-hua ${ }^{1,2}$, Yan Xiao-heng ${ }^{1}$, Peng Ji-shen ${ }^{1}$ and Zhang Yan $^{1}$ \\ ${ }^{1}$ Faculty of Electrical and Control Engineering, Liaoning Technical University \\ ${ }^{2}$ College of Mechanical Engineering, Liaoning Technical University
}

\begin{abstract}
This paper is based on Orthogonal Frequency Division Multiplexing (OFDM) technology, which is the core technology of the coal mine medium voltage electric bridge set. And to reduce the high peak-to-average power ratio arising from the OFDM signal and to strengthen the timeliness and accuracy of communication, it puts forward a real-valued genetic improvement of Partial Transmit Sequences Algorithm (RVGA-PTS) and the Timing Synchronization Algorithm which is based on SC algovithm. Finally, it completes the preliminary design of hardware system for the medium yoltage electric bridge set. The simulation results show that compared with other optimization algoxithm, the convergence speed is faster, the timing estimation precision is higher, and the system performance has been improved obviously.
\end{abstract}

Keywords: MV_PLC, Orthogonal Frequency Division Multiplexing (OFDM), Timing synchronization, Partial Transmit Sequence (PTS)

\section{Introduction}

The coal mine tunnel which is long and narrow, moves toward complex, "water, gas and dust" harm coexists, and with massive strong jamming sources, the communication environment is quite ansatisfactory compared with the ground communication in the ease and flexibility. At present when selects the wireless communication method, the signal coverage is difficult, transmission parameter is not stable and the interference noise is big. And other wired ways like the telephøne cable, fiber optics face other issues in setting line and line maintenance. This paper proposes a power line carrier (PLC) communication technology which uses the underground power line of the existing as the communication transmission channel. PLC technology is a hot topic in power system in recent years. It has many advantages, but due to the complex composition of power noise, it also has very strong instability with the change of the load [1]. To achieve fast, reliable power line data transmis 10, we must choose a modulation technique for power line channel characteristics. Introduced the orthogonal frequency division multiplexing (OFDM) technology in PLC, a line of high-speed data communication becomes possible.

OFDM technology has been used as a very effective high-speed data transfer technology and has been applied to many fields of wireless communication because of its ability of anti-multipath interference and high spectrum utilization. But the OFDM signals will bring greater peak-to-average power ratio (PAPR). The faults can make the OFDM signals distortion in nonlinear distortion of high power amplifier, leading to the BER performance degradation; This require that the power amplifier in the system sends has a large enough linear range, but this requirement in respect of hardware implementation is difficult, and the 
cost is very high [2]. Therefore, PAPR problem has become an obstacle to practical OFDM technology.

In recent years, research on PAPR reduction has become a hot issue. At present, there are some valuable methods to reduce PAPR. However, some of these methods for PAPR performance improvement are unlikely, others are higher computational complexity. These are not entirely satisfactory [3]. The first category is the signal distortion technology. It reduces PAPR through the peak power point of OFDM signals. This is one of the most simple and intuitive ways, but it increase the OFDM signal in-band interference and out-of-band radiation and bring the system serious performance loss. The second category of methods is the encoding method. Golay Code has already been used to the OFDM system with the forward error correction, and get a better lower PAPR effect. However, the flaws of this approach are that the encoding pattern number we can use is very small, especially when the number of carriers is large, coding efficiency is very low. The third category is based on the disruption of the sequence. It is mainly used to the right of disposal with different scrambled sequence of the OFDM and selects the smallest PAPR value OFDM symbol for the transfer [4]. However, larger computational complexity becomes the biggest barrier to indplementing such methods. To this end, this paper proposes a PTS algorithm which is based on real-valued genetic algorithm to improve in real-time and accuracy of the strengthening of communication. Compared with the PTS algorithm, the RVGA-PTS PAPR has the same or better performance, and the complexity is significantly reduced.

\section{The PAPR of OFDM System}

If one OFDM system containing N/sub-garriers, and during a symbolic time interval, the baseband signals can be expressed as

$$
x_{n}=\frac{1}{\sqrt{N}} \sum_{k=0}^{N-1} X_{k} e^{j \frac{2 \pi k n}{N}}, 0 \leq n \leq N-1
$$

The $\mathrm{Xk}(\mathrm{k}=0,1, \cdots, \mathrm{N}$ - $)$ is frequency domain subcarrier signal, which was got after the PSK or QAM modulation for the binary input signal. $\mathrm{Xn}$ is the output signal after the OFDM modulation, $\mathrm{n}$ is discrete time inde $\mathrm{X}$.

OFDM signal is the sum of a number of independent modulated sub-carrier signals. After adding the signal may have arelatively higher peak power, and bring about a higher PAPR. PAPR was defined as: The ratio of each peak power of OFDM signal with the average power of the same OFDM signal, that is,

$$
\operatorname{PAPR}(x)=10 \log _{10} \frac{\max _{0 \leq n \leq N-1}\left\{\left|x_{n}\right|^{2}\right\}}{E\left\{\left|x_{n}\right|^{2}\right\}}
$$

In the formula, the $\mathrm{E}\{*\}$ denotes mathematical expectation operator, $\mathrm{x}=[\mathrm{x} 0, \mathrm{x} 1, \cdots \mathrm{xN}-1] \mathrm{T}$.

For the sampling interval in the form of equation (1), we may lose some signal power peaks, because most systems use discrete-time signals. And we may mistakenly think the PAPR performance is optimal. To this end, in order to get an approximate real PAPR performance, we always introduce sampling in actual systems. $\mathrm{L}$ is the sampling factor. The implementation is added (L-1) $\mathrm{n}$ zero to the original input vectors before the inverse discrete Fourier transform (IFFT). When $\mathrm{L}=4$, and we can effectively capture all the peaks. Since the PAPR is random, the probability of more than one definite value of PAPR0 is usually used to 
estimate the statistical distribution of PAPR characteristic of the system [5]. That is a PAPR system performance. This probability is called the complementary cumulative distribution function (CCDF), which can be expressed as

$$
C C D F\left(N, P A P R_{0}\right)=P\left\{P A P R>P A P R_{0}\right\}=1-\left(1-\mathrm{e}^{-P A P R_{0}}\right)^{N}
$$

\section{Traditional PTS Algorithm}

Basic principle of traditional PTS algorithm is: Split the input length of $n$ vectors $X=\left[X_{0}, X_{1}, \ldots X_{N-1}\right]^{\mathrm{T}}$ into $\mathrm{V}$ complementary overlap block vectors $\mathrm{X}_{v}, v=1,2, \ldots V[6]$. In order to ensure the length of each character vector is $n$, make the block vectors for each word ${ }^{\bullet}$ in the left sub-carrier location data is zero, in which case the input vector $\mathrm{x}$ can be expressed as:

$$
\mathrm{X}=\sum_{v=1}^{V} X_{v}
$$

And then phase-weight each child block vectors vith weighting factors $\left\{b_{v}, v=0,1, \ldots V\right\}$, and $b_{v}=\exp \left(j \varphi_{v}\right)$. Adjust and make IFFT for $b_{v}$. Make a sum of all sub-blocks of the phase-weighted vectors and calculate the PAPR, and finally select the minimum PAPR vector for transmission. Typically, in order to avoid unnecessary calculations, adjust the process of multiplication of weighting factors $b_{v}$ and vector $\mathrm{X}_{v}$ affer the IFFT with linear nature of the IFFT transformation. As shown in Fig ire 1, the formula is as follows:

$$
y=\operatorname{IFFT}\left\{\sum_{v=1}^{v} b_{v} x_{v}\right\}=\sum_{v=1}^{V} \operatorname{IFFT}\left\{b_{v} X_{v}\right\} \Rightarrow \sum_{v=1}^{V} b_{v} \operatorname{IFFT}\left\{\mathrm{X}_{v}\right\}
$$

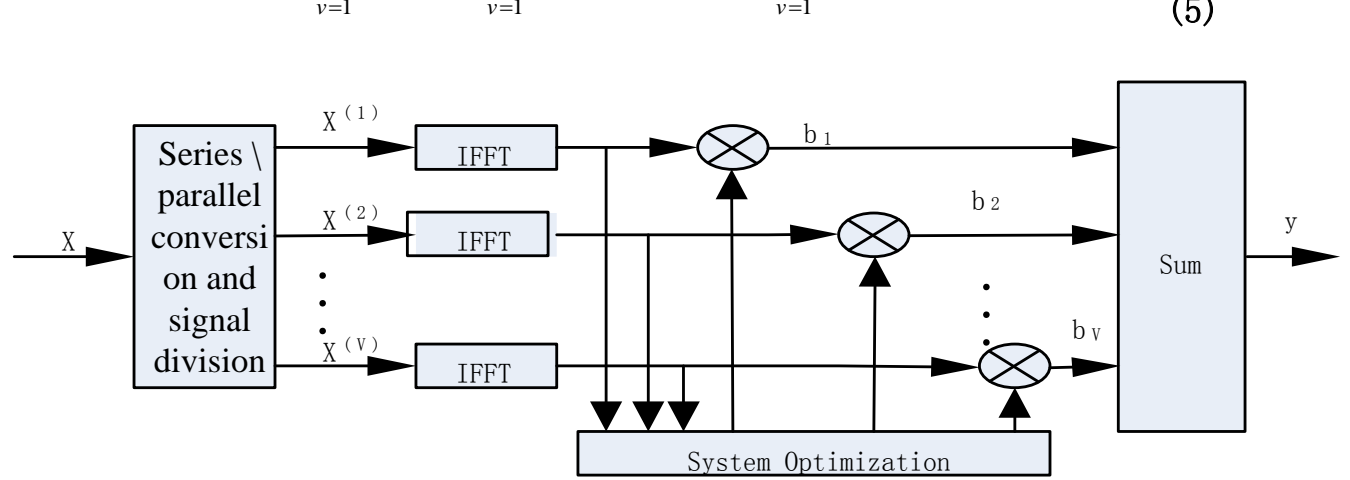

Figure 1. PTS system block diagram

The key of PTS algorithm is how to pick the best weighting factors to reduce the high peak-to-average power ratio opportunity and get the best phase sequence. To solve the method to search and optimize the phase weighting factor, the paper use genetic algorithms.

\section{RVGA-PTS Algorithm based on Immigration Policy Improvements}

This paper around the PTS algorithm study of PAPR reduction technology in probability puts forward a Kind of immigration policy to improve real-valued genetic PTS new algorithms to reduce the PAPR OFDM system. The genetic algorithms (GA) simulate the 
phenomena such as reproduction, crossover and mutation in the natural selection and heredity. Starting from any initial population, through random selection, overlapping and mutation, one crowd which is adapt to the individual of environment is created, making the community evolved to the space in better region. Such generations of unceasing multiplication, most adapts to the individual of environment restraining to one crowd finally, and obtains the issue optimal solution in the end [8].

\subsection{GA-PTS algorithm}

GA-PTS uses genetic algorithm as an alternative method to search optimal PTS phase factor, thereby reducing the PAPR of OFDM system. Compared with the traditional PTS algorithm, the maximum advantage of GA-PTS algorithm is that it improves the large quantity of calculation to a certain extent [9].

GA-PTS algorithm uses binary vectors representing the chromosome. In each chromosome, $\log _{2} W$ bits correspond to the binary representation of phase factor. If $\mathrm{W}=4$, for a group phase factor $b=[+1,-1,+j,-j]$, the correspondence betsveen the $C=$ [00110110] and $\mathrm{b}$ is: $(+1 \rightarrow 00),(-1 \rightarrow 11),(+\mathrm{j} \rightarrow 01),(-\mathrm{j} \rightarrow 10)$.

First create a population randomly. Each chromosome of the population corresponds to the phase factor, then, multiply the PTS series, we get the PAPR of computing systems. The fitness of each chromosome in the population can be expressedas:

$$
\mathrm{F}(x(t))=\frac{1}{10 \lg \operatorname{PAPR}(x(t))}
$$

Calculate of each chromosome. And with roulette method we can choose the candidate chromosomes to generate next population according the fitness [10]. After crossover and mutation, the chromosomes ane the chromosomes of next population. After largest genetic algebraic reconstruction, we canselect genome with a minimum PAPR.

\subsection{Improved RVGA PTS algorithm based on immigration policy}

When according to conventional GA-PTS operation, there will be "precociously" phenomenon, namely after certam generations the individual in copulation pond does not have any difference. The pobulation loses the multiplicity make the algorithm appear early the issue of restrain. Since then the mate to produce offspring produced no new information, and the value is only the extreme value of part, but non-overall situation extreme value. For better solution PTS-OFDM system the choice question of rotation phase factor, this paper uses the immigration strategy to solve the above problem [11]. RVGA-PTS basic block diagram of the algorithm is shown in Figure 2. Namely selects the previous generation best individual oreplace in the population each time the equal amount bad individual, and judges the genetic algorithm using the sufficiency value and population of population individual the variance of average sufficiency value whether falls into the condition that the multiplicity decreases progressively. For convenience computation, command:

$$
E=\frac{1}{N} \sum_{i=1}^{N}\left|f_{i}-f_{a v g}\right|
$$

In the formula: $f_{i}$ behalf of the sufficiency $i$ value of individual; $f_{\text {avg }}$ behalf of the average sufficiency value of community; $\mathrm{N}$ is the standard group scale. When $\mathrm{E}$ is smaller 
than some threshold value, we can think that the algorithm appeared "precociously", carries on "immigration strategy" [12].

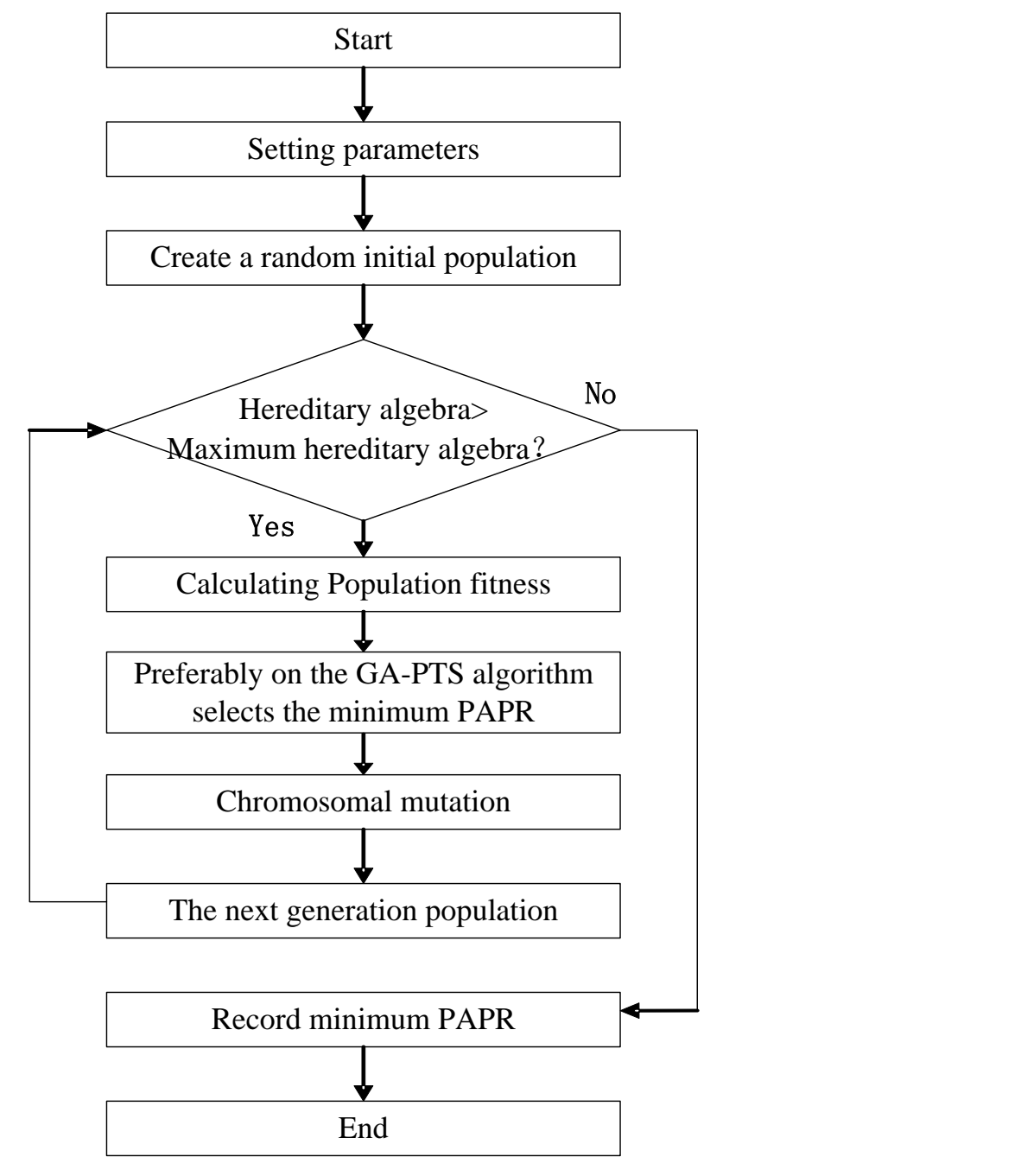

Figure 2, The basic diagram RVGA-PTS algorithm

\section{Study of Synchronization in OFDM Systems}

The synchronzation is to let the both sides of receive and dispatch marches in step on the frequency ane and phase, the synchronization quality fit and unfit quality will affect the success of information transmission directly, especially will relate to the entire communication system in the digital communication system the performance, therefore attach importance to the synchronous problem to guarantee the OFDM system transmission information the performance.

\subsection{Synchronization techniques in OFDM systems}

Every kind of synchronization and the correspondence position in OFDM system are shown in Figure 3. Figure 3 has given the OFDM system models and the location of synchronization technology in the system. 


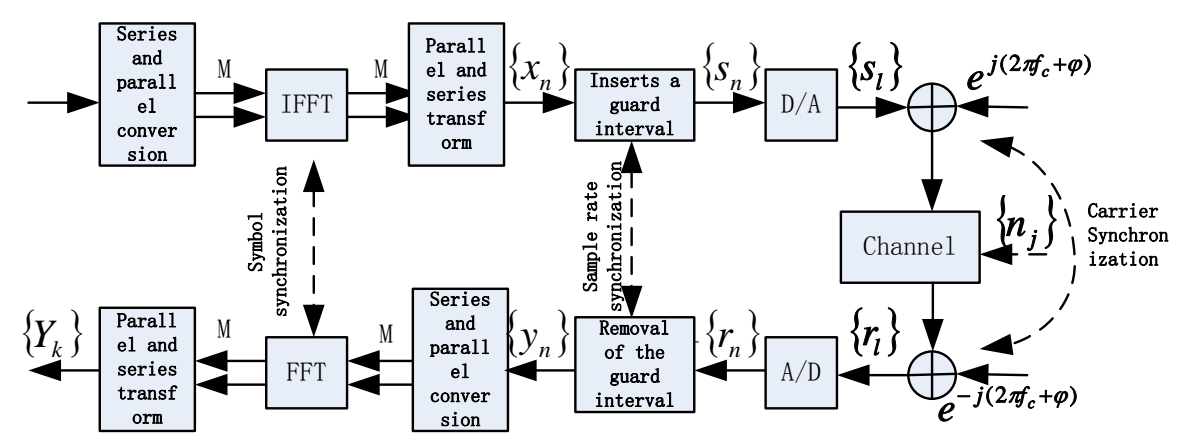

Figure 3. OFDM system synchronization

Regarding the power line transmission channel, the channel has the multi-path to pass on diameter and other characteristics, and it may make the OFDM signal code of transmission fuzzy and various sub-carrier shifts; Moreover, the deviation of the crystal oscitiator source of the transmitting end and receiving end will cause the sampling rate not to synchronize [13], therefore the actual output of FFT is:

$$
Y_{k}=X_{k} H_{k} e^{j\left(2 \pi k \frac{T_{\theta}}{T_{\mu}}+2 \pi k \frac{\Delta t}{T_{\mu}}+2 \pi n \Delta f T\right)}+I_{k}+N_{j}
$$

After analysis, we can see the synchronization in OFPM system mainly include three aspects: First, symbol synchronization; Second, carier frequency synchronization; Third, sampling rate synchronization. The deviation of FFT window causes the phase deviation of child carrier data, in extreme cases around it will gause ISI, therefore the primary mission of OFDM synchronization to obtain the carrier synchropization symbol timing synchronizes.

\subsection{SC-based algorithm of timing synchronization algorithm}

Schmid and Cox proposed carried on fixed time and method of frequency joint estimation with the use special preparation sequence, namely the SC algorithm. This algorithm has very good robustness under the multi-path fading channel [14]. But after the symbol timing deviation with the mathemat cal analysis and simulation discovered, we found that the symbol timing deviation Will cause ISI, thus it has serious influence to the system performance. Therefore, to overcome the flaw that the SC algorithm with the peak value platform is unable to complete the precise symbol timing synchronization, this paper constructs an advanced training sequence frame which is based on SC algorithm, and improves the symbol timing synchronization algorithm simultaneously.

\subsubsection{Improyed training sequence frame structure}

The advanced training sequence frame was shown in Figure 4:

\begin{tabular}{|c|c|c|c|c|c|c|}
\hline & & $\mathrm{N} / 2$ & $\mathrm{~N} / 2$ & & $\mathrm{~N}$ & \\
\hline Data symbols & $\mathrm{CP}$ & $A_{1} A_{2} \ldots A_{N / 2}$ & $A_{N / 2} \ldots A_{2} A_{1}$ & $\mathrm{CP}$ & & Data symbols \\
\hline
\end{tabular}

Figure 4. based on the SC algorithm to improve the training sequence 
After the improvement, the training sequence 1 around two parts shows the symmetric relation. In the training sequence 1 , the latter half $\mathrm{N} / 2$ sequence mark is to the central turn over of first half N/2 sequence mark. In computation of symbol timing synchronizes, the improved training sequence 1 frame structure can effectively avoid the timed peak flat area and the flaws unable to pinpoint the defect caused by cyclic prefix CP [15].

\subsubsection{Improve algorithm analysis}

The improved algorithm still uses the correlation function $\mathrm{R}(\mathrm{d})$ that defined in the SC algorithm, but the energy function makes the following improvement: Energy functions are no longer defined by the last N/2 sequences of symbols of the training sequence from the SC algorithms 1 , but defined by sequence length of the entire improved training sequence 1 , ${ }^{\bullet}$ according to this, we may define the training sequence 1 energy function :

$$
\begin{aligned}
R^{\prime}(d) & =\frac{1}{2}\left[\sum_{n=0}^{N / 2-1}|r(d+n)|^{2}+\sum_{n=0}^{N / 2-1}|r(d+n+N / 2)|^{2}\right] \\
& =\frac{1}{2}\left[\sum_{n=0}^{N / 2-1}|r(d+n)|^{2}+\sum_{n=0}^{N-1}|r(d+n)|^{2}\right] \\
& =\frac{1}{2} \sum_{n=0}^{N-1}|r(d+n)|^{2}
\end{aligned}
$$

The improved symbol timing synchronizationmeasuring function is defined as:

$$
M^{\prime}(d)=\frac{(P(d))^{2}}{(R(d))^{2}}
$$

Then the symbol timing synchronization estimated position is:

$$
\hat{d}^{\prime}=\arg \max M^{\prime}(d)^{x}
$$

There will be a sharpspike in the posjtion of symbol timing estimation, thus we can obtain the estimated location $d^{\prime}$ of symbol timing synchronization signals. Therefore in the improved algorithm, it can be very easy to find the best symbol timing synchronization point according to the kurtosis, thegaccuracy of symbol timing synchronization can greatly enhance 【16】.

In summary, the realization of improved algorithm is constituted by the following several steps:

(1) Structure the former sequence symbols of the training sequence 1;

(2) The las N/2 sequence symbols are from the overturn of the former N/2 sequence symbols, and they complete the sequence 1 structure with the length of $\mathrm{N}$;

(3) Operate the two N/2 sequences of training sequence 1, we can get the correlation to obtain correlation function $\mathrm{P}(\mathrm{d})$;

(4) Operation on training sequence 1 energy function $\mathrm{R}(\mathrm{d})$;

(5) Operation on synchronized measuring function $\mathrm{M} /(\mathrm{d})$;

(6) Obtain the best timing synchronizing estimate point, and complete the timing synchronizing. 


\section{Medium Voltage Electrical Bridge Set System Design}

This article uses DSP and FPGA as the core of the data processing, and it may result in Figure 5 to press medium voltage electric bridge set collection basically to realize the hardware diagram based on OFDM under according to the above chart 1, the entire hardware system may be divided into the following several parts:

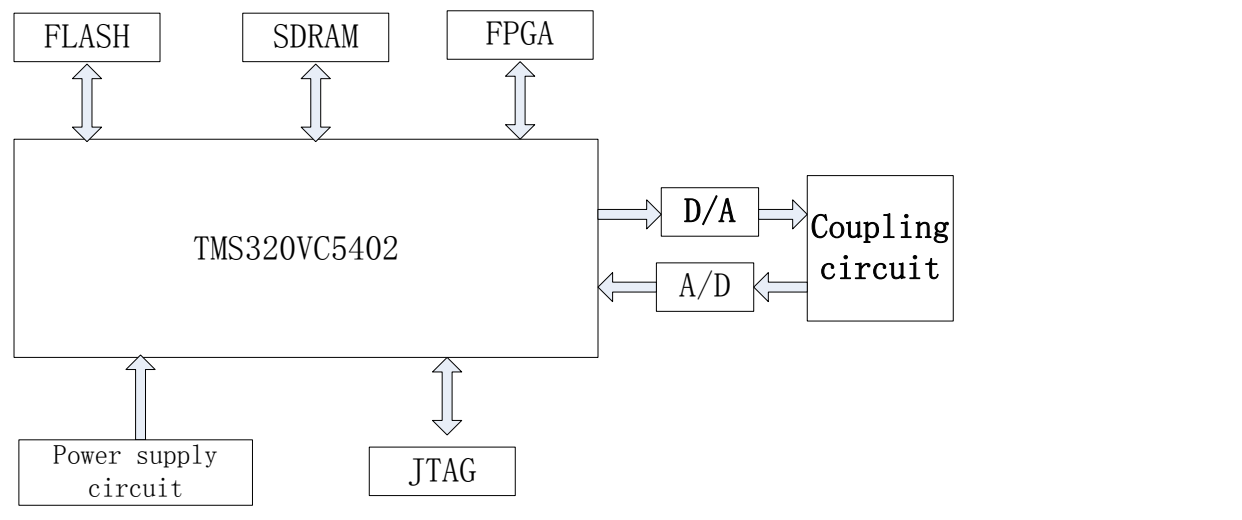

Figure 5. Based on OFDM power bridge set basic hardware frame

(1) DSP and FPGA module

The systematic DSP (DSP) uses the TMS320VC5402 position processor and controller in TI Corporation high performance fixed point DSP C54 series [17]. This module mainly realizes the following function: Koice, remote and guard signal the group frame of filter, modulation and demodulation as well as data; The production and MODEM of QPSK signal; FFT and IFFT implementation: The computation and the sub-channel choice signal-to-noise ratio SNR and reduces realization of PARPJalgorithm; Realization of synchronized algorithm.

(2) ADC and DAC module

ADC and DAC performance is affecting the whole system performance index to a certain extent. High-frequency DA Connects with DSP MCBSP (McBSP1). The initialization to McBSP1 by DSP realizes the serial communication of DSP.

(3) FLASH

When DSP is on electricity, FLASH with the DSP EMIFB CE1 space connects will realize the BOOTLOADER function, namely the code information in FLASH will change over to DSP RAM automatically, after that, DSP starts the executive routine from the procedure entry point address.

(4) SDRAM

SDRAM is synchronous dynamic random access memory. When the procedure and data capacity are bigger than DSP interior RAM, it can store in the massive data messages SDRAM.

(5) JTAG emulation online connection 
JATG is the joint test work team abbreviation, one boundary scan method based on IEEE1149.1 standard. Ti provides JTAG port support for most DSP [18]. It has provided a real-time hardware simulation and debugging environment for the development staff.

\section{Algorithm Performance Simulation Results and Analysis}

\subsection{Simulation analysis of MRVGA-PTS algorithm}

In the numerical simulation, we suppose the OFDM system sub-carrier integer $\mathrm{K}=128$, with the QPSK modulation, 4 times of over samplings, phase factor weight $\mathrm{W}=4$, namely the phase set is $b_{v}=\{ \pm 1, \pm j\}$. With the neighboring division, the computer selects 100000 independent OFDM marks stochastically, the supplementary distribution function $\mathrm{CCDF}=0.1 \%$. The crossover probability $\mathrm{PC}=0.5$, heredity algebra $\mathrm{G}=30$, the population scale counts $\mathrm{P}=300$, sub-carrier division block nymber $\mathrm{V}=10$, mutation probability $\mathrm{Pm}=0.08$. When the immigration strategy ebpyation pond has not been carried out, the scale count $\mathrm{T}=15$, but when works $\mathrm{T}=5$ [19].

Optimize the phase factor with the MRVGA-PTS algorithm. Then, we obtain the minimum system PAPR value, and get its PAPR simulation CCDF statistical curve, and obtain the CCDF statistical curve as shown in Figure 6 after comparing with exhausting optimum algorithm (Exhaustive) and diffractive opfical element (SA-PTS) and traditional binary coding genetic algorithm (BGA-PTS) and iterative then over algorithm (IF-PTS) compares. When $\mathrm{CCDF}=0.1 \%$, the PAPR valued of OFDM system is: $5.85 \mathrm{~dB}$ to exhausts search method, $5.92 \mathrm{~dB}$ to RVGA algorithm, $6.05 \mathrm{~dB}$ to $\mathrm{SA}$ algorithm, $6.13 \mathrm{~dB}$ to BGA algorithm, $7.68 \mathrm{~dB}$ to IF algorithm and $10.68 \mathrm{~dB}$ to the primrive OFDM signal. The result demonstrates that the systematic PAPR value of the MRVGA algorithm designed by this article only misses $0.07 \mathrm{~dB}$ compared with the exhausting search method, but the computation load is $6.05 \%$ of the latter. The compares of every searching revolving phase factor plan are shown in Table 1, When concerning BGA, the restraining is slow, and the restraining effect was inferior to the MRVGA algorithm under the same computation load condition; Computing complexity of IF algorithm is lowest, but the performance is inferior; Computing complexity of exhausts the searching algorithm is highest, but the effect is best; Performance of BGA and SA algorithm is better to IF, and they have a quicker convergence rate, but a higher complexity than IF; The MRVGA algorithm restrains a best effect compared with other sub-optimization algorithm. Its convergence rate is faster, and the computation load is the same as SA and BGA algorithm. 


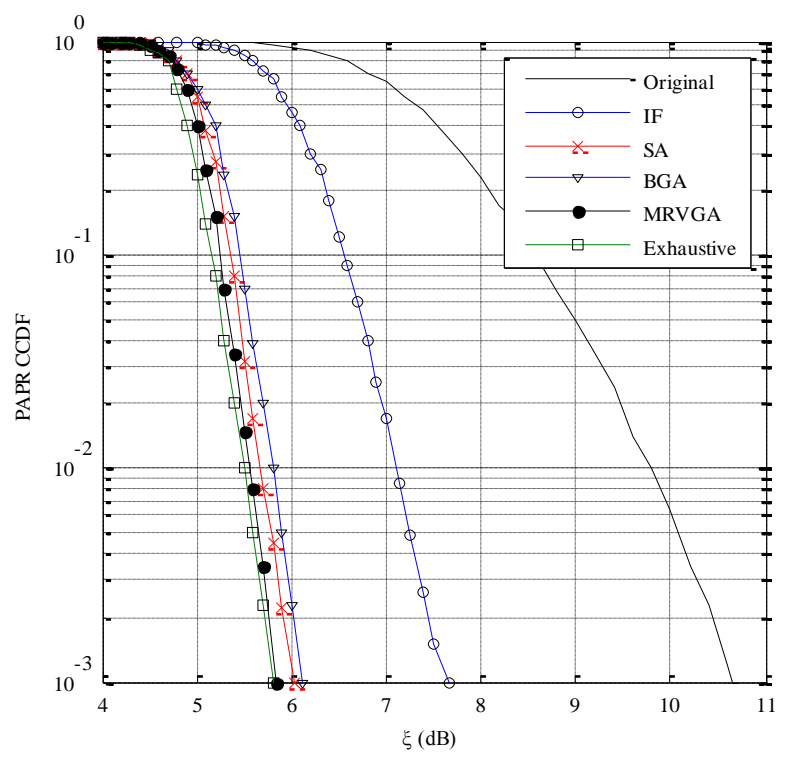

Figure 6. Algorithm OFDM signal complementary cumulative probability distribution simulation results

Table 1. Search rotary phase factor scheme comparison

\begin{tabular}{|c|c|c|}
\hline Method & Number of search & $\xi(\mathrm{N}=128)$ \\
\hline Exhaust method & & 5.85 \\
\hline IF & $(M, 1) \times W$ & 7. 68 \\
\hline SA & 4, 000 & 6.05 \\
\hline BGA & $P \times G$ & 6.13 \\
\hline MRVGA-E & $P \times G$ & 5.92 \\
\hline
\end{tabular}

To confirm the MRVGA-PTS algorithm performance further, Figure 5 measures the difference of sub-optimization algorithm in PAPR reduction performance and computing complexity. The $\mathrm{Y}$ coordinate represents the system $\mathrm{PAPR}$ value when $\mathrm{CCDF}=0.1 \%$, the abscissa corresponds to number of searches. When searching 100 times, the difference of performance of traditional genetic algorithm and improvement real value genetic algorithm is not very obvious, and most PAPR value can be controlled below $6.8 \mathrm{~dB}$, but the method of simulated annealing perform deviate. The search capabilities of simulated annealing algorithm and real-valued genetic algorithm have gradually manifested with the number of search increase, as shown in Figure 7, when search 1000 times, convergence of the MRVGA-PTS and SA-PTS is faster, and the effect restrains better to the BGA-PTS algorithm gradually. It proofs that the performance of MRVGA-PTS algorithm is better than other PTS sub-optimization algorithm. 


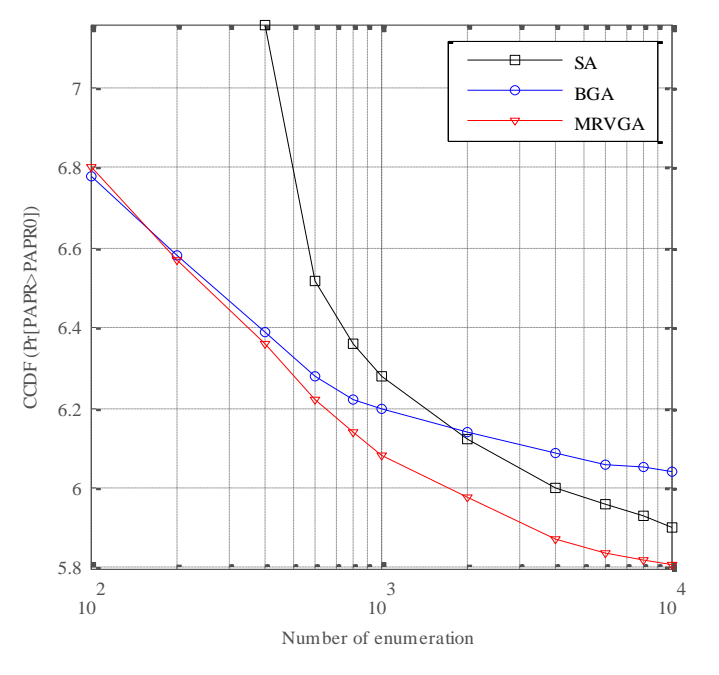

Figure 7. Search times and reduced PAPR performance relationship

\subsection{SC-based algorithm for simulation and analysis of timing synchronization algorithm}

In the synchronization, the quality of synchronous capability affects the signal the size of error rate directly; therefore we can weight system synchronous capability with error rate. With the MATLAB simulation method, we can simulate the timing synchronizing performance of the OFDM system from the timing synchronizing measuring functions and error rate two aspects with improved algorithm designs [20]. With the QPSK modulation system in the simulation system, the OFDM systematic sub-carrier counts $\mathrm{N}=256$, the length of cyclic prefix is $c p=32$. In order to verify the impact of improved algorithm to measure timing synchronization function, Be simulate the system in ideal environment free from noise and signal-to-noise ratio of white Gaussian norse channels SNR=10dB. Figure 8 and 9 are separately the timing synchronizing measuring function $\mathrm{M}$ (d) curve of the SC algorithm and the improved algorithm.

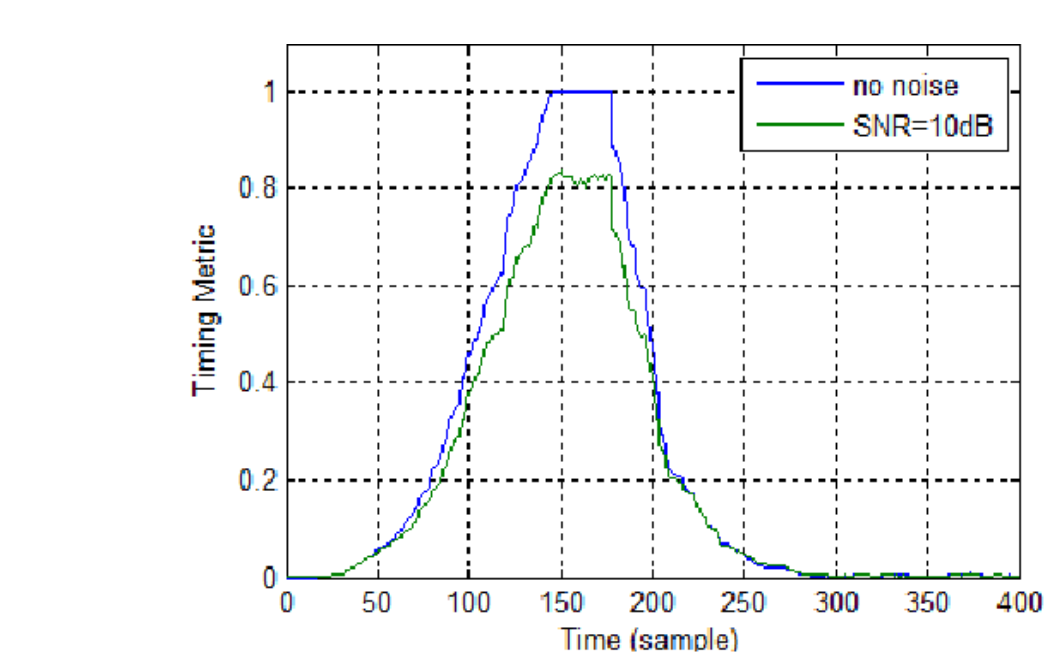

Figure 8. SC algorithm and $\mathrm{SNR}=10 \mathrm{~dB}$ ideal case timing metric simulation diagram 
From the simulation result compare Figure 9 with Figure 10, we can see that: Improved algorithm eliminate the peak platform effectively in the position symbol timing synchronization caused by cyclic prefix in SC algorithm. There are spike waveforms in the place of the correct symbol timing, and it can fulfill symbol timing estimation based on the sharp peak and end symbol timing synchronization. The improved algorithm enhances the precision of the symbol timing synchronization.

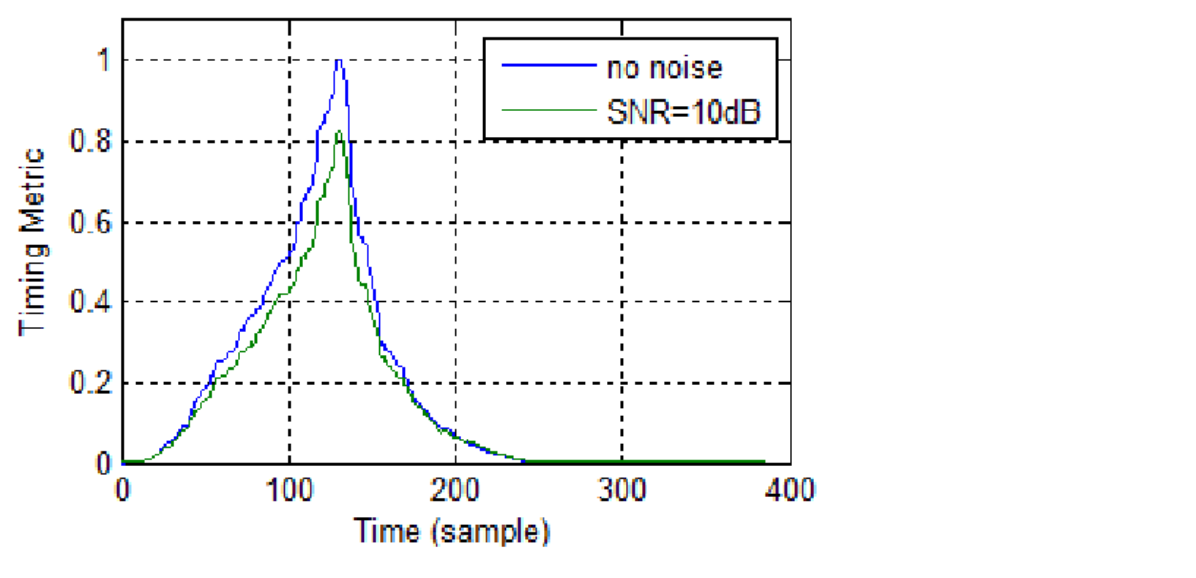

Figure 9. Improved algorithm ideal and $S N B=10 \mathrm{~dB}$ cases set metric function simulation diagram

Figure 10 is the comparison of SC and improved algorithms from both error rate and signal to noise ratio in AWGN channel environment. The abscissa represents the system signal-to-noise ratio; the $\mathrm{Y}$ coordinate represents the corresponding system the error rate. From Figure 10 we may know that the improved algorithm obtains a big reduction in error rate compared with the original algorithm under the condition of same signal-to-noise ratio. Moreover, with increase of signal-to-noise ratio, the superiority of the improvement algorithm is getting more obvious, and the system performance is significantly better than the original algorithm.

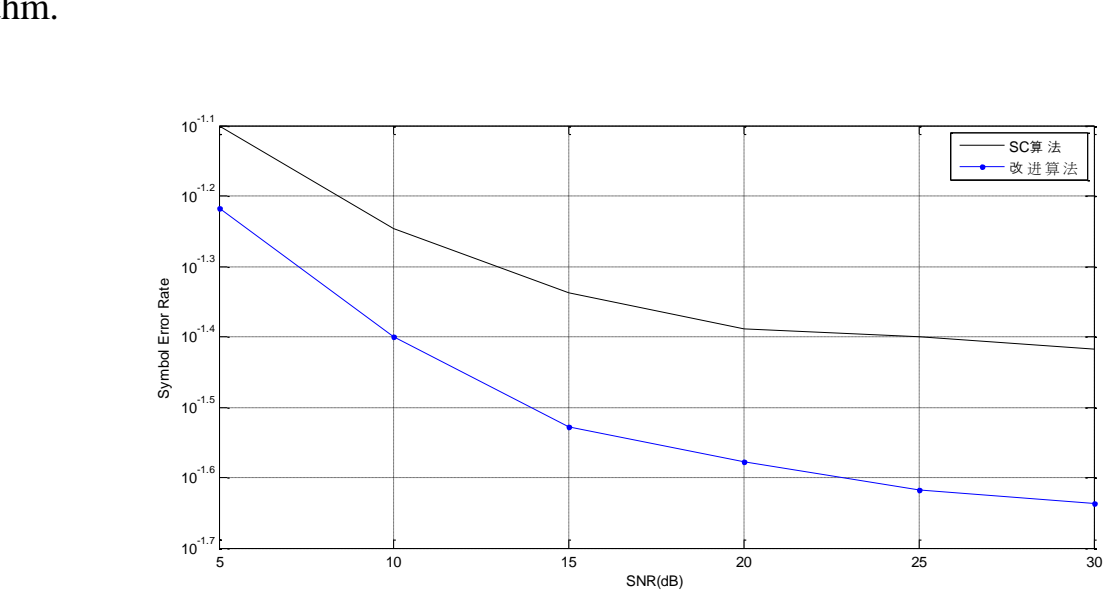

Figure 10. SC algorithm with the improved algorithm of symbol error rate simulation 


\subsection{Analysis of OFDM simulation platform design}

This paper constructs the entire OFDM system with MATLAB referring to Figure 1. It includes the signal data production, the MODEM, the communication channel simulation, the channel coding, the symbol timing synchronization algorithm, the PAPR algorithm to reduce PTS to conduct the software simulation and performance test.

Figure 11(a to d) presses several of simulation models and module structure drawings for the medium voltage electric bridge set of OFDM system. The simulation platform is mainly comprised of the OFDM transmitting end, the channel and OFDM receiving end.
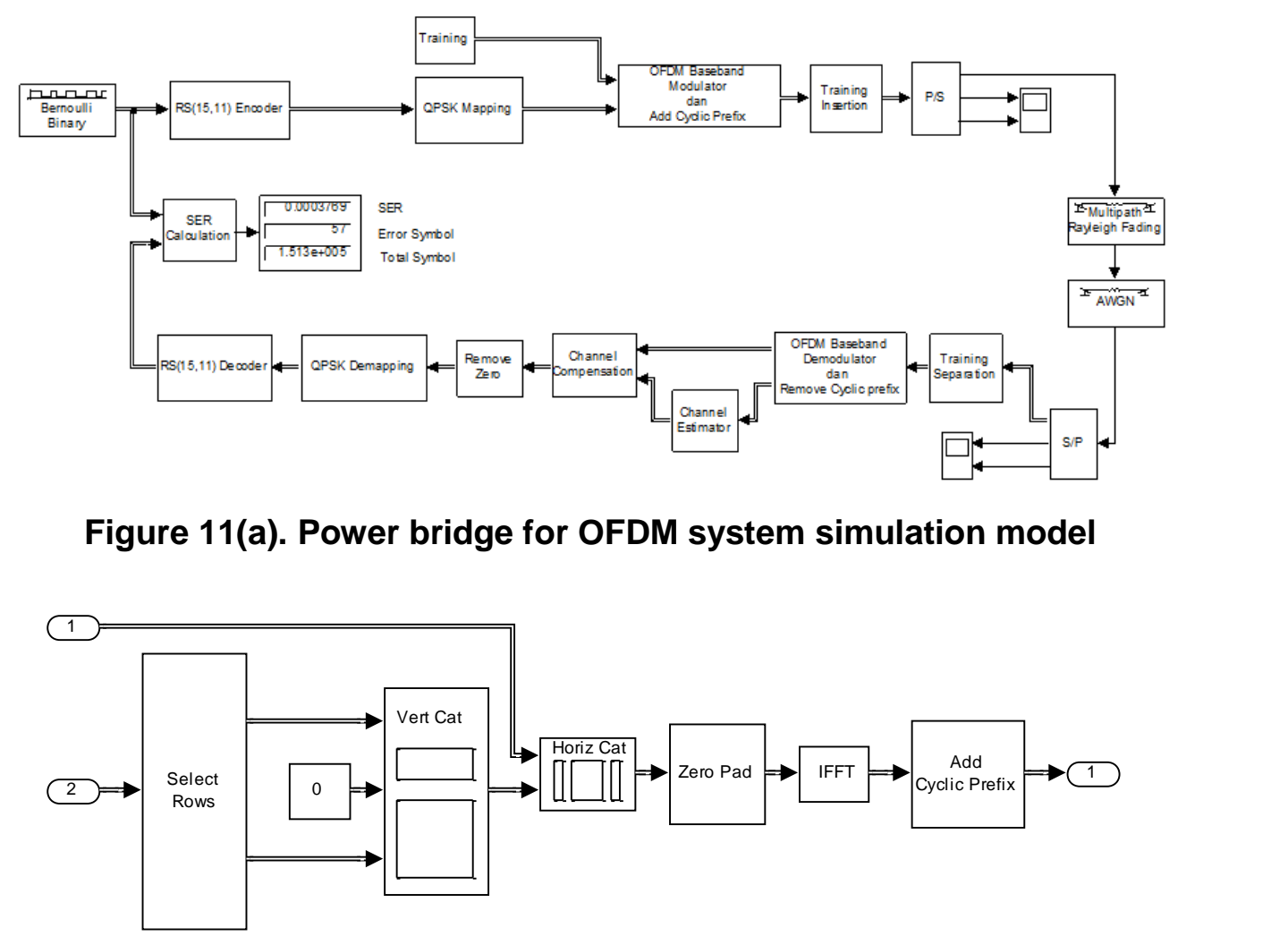

Figure 11(b). The OFDM module structure of the system

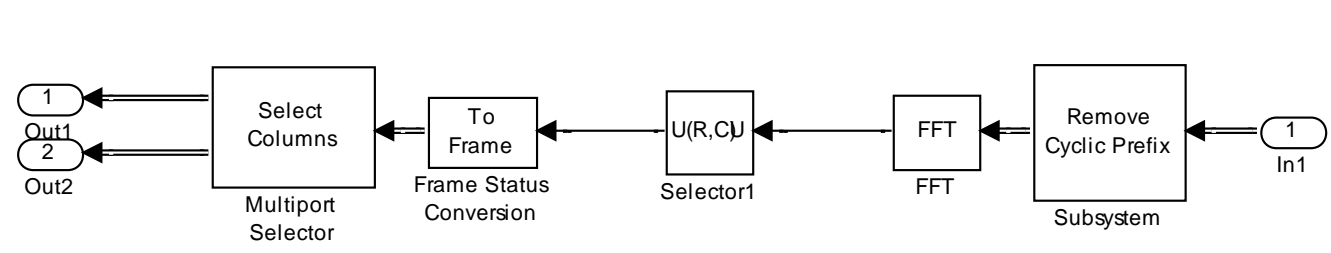

Figure 11(c). The OFDM demodulate structure of the system 


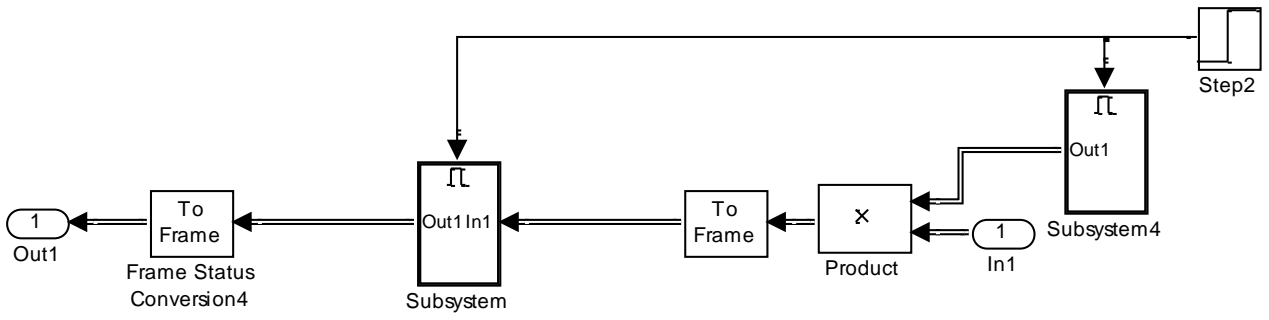

Figure 11(d). Channel estimation module structure

The transmitter modules are as follows:

(1) Data generation module: Generate switch quantity signals randomly with Bernoulli's signal generators. The high and low signal probability is the samer

(2) Binary RS code module: Use $(11,15)$ code.

(3) QPSK module.

(4) Cyclic prefix module.

(5) OFDM modulation module: The sub-carrier count is $64, C P-7 / 4$, namely $C P=16$, IFFT and FFT in the model can be freely set.

(6) Training sequence module.

(7) Parallel port serial converter modure.

The receiver modules are as follows:

(1) Serial and parallel port converter module.

(2) OFDM demoduation module.

(3) Channel estimation module. With the same frequencies set of the PN sequence generator and polynomial generation, poducing the same sequence can be guaranteed.

(4) Channel compensation module.

(5) Error rate surveillancé module.

Based on PTS algorithm simulation of real-valued genetic algorithm, we can determine this simulation platform have a certain using value. It can bring about the simulation optimization to the system algorithm in effectively in research of medium voltage electric bridge set.

\section{Conclusions}

This paper has profoundly studied the OFDM-based PLC system. The specific research content includes:

(1) This paper has analyzed the problem of the high peak-average ratio in OFDM system and proposed a modified PTS algorithm based on real-valued genetic algorithm, and 
confirmed the correctness of the algorithm at last through the MATLAB simulation experiment system.

(2) The paper has conducted the meticulous research to the synchronous problem of OFDM system, analyzed many effects of synchronous problems on the system, and proposed SC algorithm-based timing synchronizing improved algorithm. At last, we conducted the characteristics simulation of the improved key technology algorithms with the MATLAB, and confirmed the accuracy of algorithm.

(3) Finally, the paper has designed and constructed the whole medium voltage electric bridge set system.

The design of system for Medium voltage electric bridge set was initially completed in the simulation and design of key technologies in this paper, but many issues still remain unresolved, such as estimate algorithm research to PLC channel, the PCB board design as well as the whole system performance.

\section{References}

[1] Y. Zhang, X. Zhang and Y. Zhang, "The design of coal mine PLCC system and realizes", The 13th national youth communication conference proceedings (Part two), (2008), pp. 1353-1355

[2] J. Lu and W. Wen, "The genetic algorithm reduces the OFDM system PAPR", Guangdong communication technology, vol. 9, (2008), pp. 53-56.

[3] C. Sun, "Research and implementation of power line communication Based on OFDM System", Tianjin University College Master's degree thesis, (2006)

[4] S. Qi, "Power line communications (PLC) technologies and applications", Beijing: Chinese Electric Power Industry Publishing House, vol. 8, (2005).

[5] J. Zhou, Z. Jia and X. Tan, "A PTS OFDM PAPR reduction technique”, Communication technology, vol. 43, (2010), pp. 31-32.

[6] D. Zheng and Z. Guo, "Research on the techniques of education PAPR in OFDM system", Modern Electronics technique, vol. 23, no.28, (2005), pp) 1013.

[7] Y. Ronghua, "Reduce the Seatching algorithm of OFDM system PAPR to study", The Lanzhou University master's thesis, (2010) September.

[8] J. Lu and W. Wen "Reduce in the OFDM system the PAPR sub optimization PTS algorithm", Communication, vol. 41, (2008), pp. 14-16

[9] Y. Zhang, J. Yang and X. Lu, "Corbine high quality adaptive genetic algorithm for policy and immigration policy", Computer engineering and applications, vol. 46, no. 31, (2010), pp. 36-38.

[10] W. Zhou, Y. Cai and H. Chen Real value multi-population genetic algorithm for dynamic programming problems", Control engineering vol. 5, (2007).

[11] D. Zhang, "DSP-based L-PLC modem design", Hebei University master's thesis, (2011).

[12] S. S. Kim, M. J. Kim and T. A. Gulliver, "PAPR reduction of OFDM signals using genetic algorithm PTS technique", IEICE TRANSACTIONS on Communications, vol. E91-B, no. 4, (2008), pp. 1194-1197.

[13] L. Yang, R. S Cherr, Y. M. Siu, et al., "PAPR reduction of an OFDM signal by use of PTS with low computational complexity", IEEE Transactions on Broadcasting, vol. 52, no. 1, (2006), pp. 83-86.

[14] KIM H J, CHO S C, OH H S, et al. Adaptive clipping technique for reducing PAPR on OFDM systems [C]/2003 IEEE 58th Vehicular Technology Conference. [S. 1.]: IEEE, 2003.

[15] H. Chen and H. Liang, "A Modified Selective Mapping with Block Coded Modulation for PAPR Reduction in OFDM Systems", International Journal of Electrical Engineering, vol. 13, no. 4, (2007), pp. 331-338.

[16]L. Wang and Y. Cao, "Sub-optimum PTS for PAPR reduction of OFDM signals", Electronics Letters, vol. 44, no. $15,(\mathbf{2 0 0 8})$, pp. $921-922$.

[17] B. H. Alhasson and M. A. Matin, "PAPR distribution analysis of OFDM signals with partial transmit sequence", Proceedings of 14th International Conference on Computer and Information Technology Dhaka, Bangladesh, (2011), pp. 652-656.

[18] J. Tao and Y. Y. Wu, "An overview: peak-to-average power ratio reduction techniques for OFDM signals", IEEE Transactions on Broadcasting, vol. 54, no. 2, (2008), pp. 257-268.

[19] S. -S. Eom, H. Nam and Y. Ko, "Low-complexity PAPR reduction scheme without side information for OFDM systems”, IEEE Transactions on Signal Processing, vol. 60, no. 7, (2012), pp. 3657-3669. 
[20] H. Li, T. Jiang and Y. Zhou, "A novel sub block linear combination scheme for Peak-to-Average power ratio reduction in OFDM systems", IEEE Transactions on Broadcasting, vol. 58, no. 3, (2012), pp. 360-369.

Fund project: The Innovation team project in Education Department of Liaoning Province (LT2010047); Talents support project for colleges and universities in Liaoning Province (2008RC25); The power electronics and power transmission provincial key discipline scientific research fund project of Faculty of Electrical and Control Engineering, Liaoning Technical University.

\section{Authors}

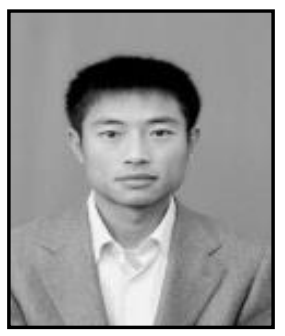

Wei-hua Chen. He is a PhD student major in Mechanical Design and Theory in College of Mechanical Engineering, Liaoning Techínical • University. Now, he is a lecturer working in the Department of Automation, Faculty of Electrical and Control Engineering of Liaoning Technical University. His current research interests are Industrial automation, etc.

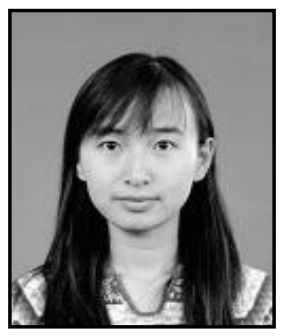

Xiao-heng Yan. Now, she is alecturer working in the Department of Automation, Faculty of Electrical and Control Engineering of Liaoning Technical University. He̊r current research interests are production process automation monitoring system. $Q$

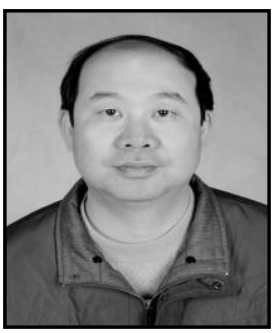

Ji-shen Peng. Now he is a Doctoral Advisor in College of Mechanical Engineering, Liaoning Technical University. His current research imterests are computer control technology and simulation of electric drive system, Mechanical design and intelligent control, etc.

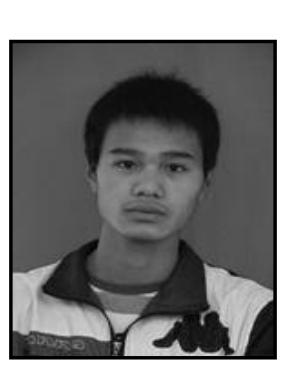

Yan Zhang. Now, he is a student working in Electrical Engineering and Automation, Faculty of Electrical and Control Engineering of Liaoning Technical University. 